\title{
Collagen breakdown during acute lung injury
}

\author{
IAN Y R ADAMSON, GILLIAN M KING, DRUMMOND H BOWDEN \\ From the Department of Pathology, University of Manitoba, Winnipeg, Canada
}

ABSTRACT Injury to the capillary endothelium and to alveolar epithelial cells of the lung may result in damage to the underlying collagen of the extracellular matrix. To examine this possibility, wholee body irradiation, bleomycin injections, and exposure to hyperoxia were used to induce various types of lung damage in mice. The morphology of the lung and the cellular and protein content of bronchoalveolar lavage fluid were used to assess injury. Collagen breakdown was assessed from the hydroxyproline concentrations in bronchoalveolar lavage fluid. When lung cell injury was observed, protein leaked in to alveoli and hydroxyproline was detected in bronchoalveolar lavage fluid. Ano increase in hydroxyproline followed endothelial damage by irradiation and was greatly increased when type 1 epithelial cell necrosis also occurred after bleomycin injection or hyperoxia. Maximak concentrations of hydroxyproline occurred in mice showing respiratory distress after six days of hyperoxia. Concentrations returned to zero during the subsequent phases of cell regeneration and $\overrightarrow{0}$ fibrosis seen after bleomycin injection and irradiation. There was little change in the cellularo components of bronchoalveolar lavage fluid at any time. The results indicate that collagen breakdown occurs during acute lung injury and can be quantified in terms of the hydroxyprolines concentration in lavage fluid. Such a change in the extracellular matrix might influence the subsequent division and differentiation of regenerating cells during repair.

\section{Introduction}

The epithelial and endothelial cells of the alveolar wall are susceptible to injury by many airborne and blood borne agents.' Focal acute injury may be rapidly repaired by proliferation of surviving type 2 epithelial and endothelial cells, which divide and spread over the underlying matrix to restore the normal structure to the alveolar wall. ${ }^{23}$ Nevertheless, as the properties of cell division, differentiation, and migration are known to vary with the underlying substrate for various cell types, ${ }^{4-6}$ severe lung injury could possibly alter the extracellular matrix, so changing the pattern and properties of cells that regenerate during repair.

In the initial stages injury to alveolar cells is accompanied by an inflammatory response, with a change in permeability that can be monitored by quantifying the cellular and fluid exudate on the basis of bronchoalveolar lavage fluid..$^{7-9}$ If injury is more severe, damage to basement membranes and connective tissue may occur and collagen fragments be released into the air spaces, where they could be detected in the bronchoalveolar lavage fluid. To

Address for reprint requests: Dr I Adamson, Department of Pathology, University of Manitoba, 236-770 Bannatyne Avenue, Winnipeg, Canada. R3E 0W3

Accepted 28 March 1988 investigate whether damage to the extracellular matrix $\overrightarrow{\overrightarrow{0}}$ occurs in acute lung injury, we used three models to induce varying degrees of damage to the pulmonary endothelium and epithelium. Mice were exposed to. whole body irradiation, hyperoxia, or intravenous bleomycin and the degree of injury to the air-bloocto barrier, assessed from the morphology and protein leakage, was related to changes in hydroxyproline concentrations in bronchoalveolar lavage fluid. As changes in extracellular collagen could be due to the direct effects of exposure to the stimulus or secondary to recruitment of inflammatory cells, we also examined the cellular and lysosomal content of the lavage fluid.

\section{Methods}

We used male, Swiss-Webster mice weighing $25 \mathrm{~g}$ in groups of four; all were killed by barbiturate overdose. They were subjected to the following three wello established models of inducing acute lung injury.

Irradiation Mice housed in individual sections of a plastic box received $9 \mathrm{~Gy}(900 \mathrm{rad})$ of whole bodyo irradition and were killed at intervals up to 12 weeks. ${ }^{10}$ Starting two weeks before exposure, all animals received chlortetracycline in drinking water through $\frac{\mathbb{8}}{2}$ out the experiment to reduce infection rates ando mortality, which was about $15 \%$. Caged control mice 
were treated similarly and killed at the same times.

Bleomycin Mice were injected intravenously with a single dose of bleomycin $(120 \mathrm{mg} / \mathrm{kg})$ in 0.1 saline and were killed up to six weeks later." Control mice received a saline injection and were killed at the same time.

Hyperoxia Animals were exposed to $95 \%$ oxygen in a plastic chamber for two, four, or six days. ${ }^{23}$ After six days, when about $20 \%$ of the mice had died, survivors were returned to room air for up to six weeks. Control animals were maintained in air only.

\section{BRONCHOALVEOLAR LAVAGE}

A tracheotomy was performed on each animal and the lungs were lavaged four times with $1 \mathrm{ml}$ normal saline. In each case the total volume recovered ranged from 3.1 to $3.5 \mathrm{ml}$. The total cell count was determined with a haemocytometer and a cytocentrifuge preparation was made for differential counts of alveolar macrophages and polymorphonuclear leucocytes. The lavage fluid was centrifuged and frozen for later analysis. Total protein concentration, ${ }^{12}$ glucosaminidase activity, ${ }^{13}$ and hydroxyproline concentration ${ }^{14}$ were determined.

After the lungs had been lavaged, the right bronchus was clamped and the right lung used for measuring tissue hydroxyproline. The other lobes were inflated with buffered glutaraldehyde and samples prepared for electron microscopy.

\section{STATISTICAL ANALYSIS}

The means and standard errors of all measurements were calculated at each time interval and differences between experimental and control groups were examined by Student's $t$ test for significance. For simplicity of presentation the overall means for all controls in each experiment are shown in the tables.

\section{Results}

\section{IRRADIATION}

Morphological examination of the lung showed evidence of injury to capillary endothelial cells up to two weeks after irradiation. Many cells showed vacuolation of the cytoplasm and focal areas of interstitial oedema were seen (fig 1). No injury was seen in other cell types; the epithelial surface was normal and no inflammatory cells were found in the lung. After two weeks the endothelium appeared normal and there was no evidence of fibrosis until eight weeks, when focal thickening of alveolar walls was seen.

The total number of cells recovered by lavage did not differ between the treated and the control animals; no change in macrophage or leucocyte counts was seen during the experiment (table 1). A significant increase

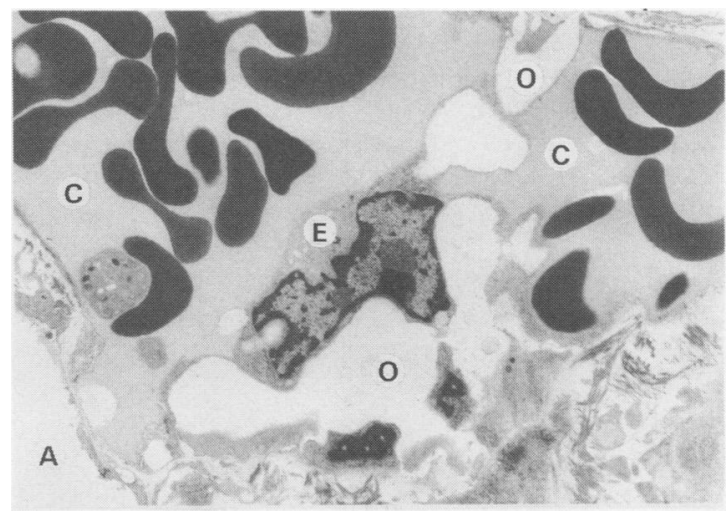

Fig 1 Injury to endothelial cells, seven days after whole body irradiation, resulting in cytoplasmic swelling and areas of subendothelial oedema $(O)$. C-capillary; $A$-alveolar space; $E$-endothelial cell.

in glucosaminidase activity was found one week after exposure (table 1) and total protein was slightly increased at 10 days, but otherwise was within the normal range (fig 2). Hydroxyproline was never detected in bronchoalveolar lavage fluid from normal animals; the limit of detection in our assay was 0.001 $\mu \mathrm{g} / \mathrm{ml}$ bronchoalveolar lavage fluid. One week after irradiation a peak level of hydroxyproline of $0.1 \mu \mathrm{g}$ was found; values had returned to near zero after four weeks (fig 2). During this time there was no significant change in total hydroxyproline in lung tissue, but after four weeks, as fibrosis developed, the concentration of hydroxyproline per mg dry lung was significantly greater than that of age matched controls (fig 3 ).

\section{BLEOMYCIN}

The morphology of mouse lung after intravenous bleomycin has been described. ${ }^{7}$ At five days there was endothelial injury, which was more extensive than that seen after irradiation, and by 10 days there was focal necrosis of type 1 epithelial cells. Repair was under way by three weeks, with proliferation of type 2 cells

Table 1 Findings in bronchoalveolar lavage fluid after irradiation

\begin{tabular}{llll}
\hline $\begin{array}{l}\text { Time after } \\
\text { irradiation }\end{array}$ & $\begin{array}{l}A M \\
\left(\times 10^{4}\right)\end{array}$ & $\begin{array}{l}P M N \\
\left(\times 10^{4}\right)\end{array}$ & $\begin{array}{l}\text { Glucosaminidase } \\
(\mu \mathrm{mol} / \mathrm{ml} / \mathrm{h})\end{array}$ \\
\hline [Controls & $20(4)$ & 0 & $100(12)]$ \\
$3 \mathrm{~d}$ & $21(5)$ & 0 & $120(17)$ \\
$7 \mathrm{~d}$ & $21(3)$ & 0 & $182(22)^{*}$ \\
$2 \mathrm{w}$ & $19(3)$ & 0 & $130(18)$ \\
$4 \mathrm{w}$ & $21(4)$ & $0 \cdot 1(0 \cdot 1)$ & $135(30)$ \\
$8 \mathrm{w}$ & $22(4)$ & $0 \cdot 1(0 \cdot 1)$ & $148(27)$ \\
$12 \mathrm{w}$ & $25(3)$ & $0 \cdot 1(0 \cdot 1)$ & $125(25)$ \\
\hline
\end{tabular}

${ }^{*} \mathrm{p}<0.01$ in the comparison with controls.

AM-alveolar macrophages; PMN-polymorphonuclear leucocytes. 


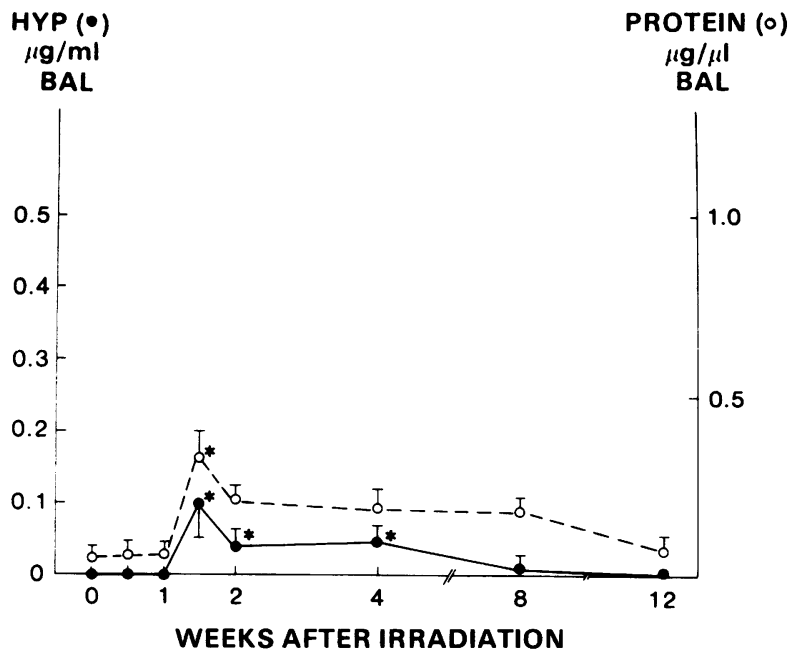

Fig 2 Hydroxyproline (Hyp, closed circles) and protein (open circles) concentrations in bronchoalveolar lavage (BAL) fuid after whole body irradiation. ${ }^{*}$ Values are significantly higher than for age matched controls at $p<0 \cdot 01$.

and fibroblasts. By six weeks the fibrotic process was more obvious.

There was no change in the numbers of alveolar macrophages recovered by bronchoalveolar lavage but there was an increase in polymorphonuclear leucocytes at the times of maximal cell injury (table 2 ). The total number of polymorphonuclear leucocytes was $1 \times 10^{4}$ and the increase was accompanied by an increase in glucosaminidase activity at five days. Protein concentrations in bronchoalveolar lavage fluid were also significantly greater than in control lavage fluid at five and 10 days (fig 4). During this period there was a substantial increase in hydroxyproline to $0.3 \mu \mathrm{g} / \mathrm{ml}$ fluid and this was still greater than control values at three weeks (fig 4). Hydroxyproline concentrations in tissue were unchanged during this period but increased later as fibrosis developed (fig 3).

\section{OXYGEN}

Prolonged hyperoxia produced the most severe lung cell injury. After two days in $95 \%$ oxygen mild endothelial injury was seen, and by day 4 injury to this cell type was more diffuse and many vacuolated cells with underlying oedema were seen. At this stage cell injury was confined to the endothelium, but by day 6 some animals were in respiratory distress. Many type 1 epithelial cells were necrotic, leaving a denuded bloodair barrier (fig 5); and in severely injured animals polymorphonuclear leucocytes were often found sequestered in capillaries. The $20 \%$ of mice who died had solid lungs. The survivors who were returned to

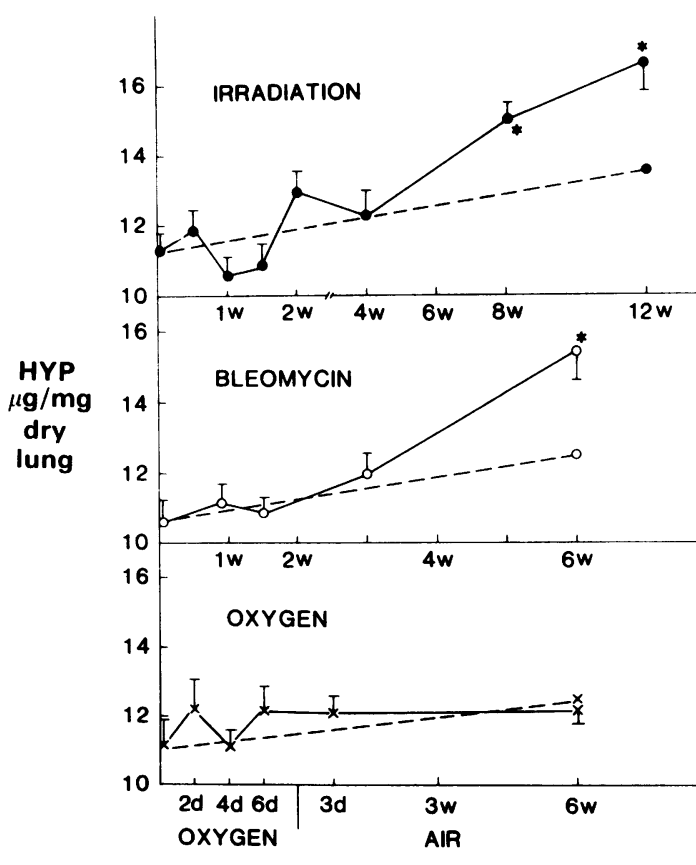

TIME AFTER TREATMENT

Fig 3 Hydroxyproline ( $\mathrm{Hyp}$ ) content of lung tissue after irradiation (closed circles), bleomycin (open circles), and oxygen (crosses). Control concentrations are shown by the broken lines. ${ }^{*}$ As in figure 2. 
Table 2 Findings in bronchoalveolar lavage fluid after bleomycin injection (means with standard errors in parentheses)

\begin{tabular}{llll}
\hline $\begin{array}{l}\text { Time after } \\
\text { injection }\end{array}$ & $\begin{array}{l}A M \\
\left(\times 10^{4}\right)\end{array}$ & $\begin{array}{l}P M N \\
\left(\times 10^{4}\right)\end{array}$ & $\begin{array}{l}\text { Glucosaminidase } \\
(\mu \mathrm{mol} / \mathrm{ml} / \mathrm{h})\end{array}$ \\
\hline [Controls & $17(4)$ & $0 \cdot 1(0 \cdot 1)$ & $120(16)]$ \\
$5 \mathrm{~d}$ & $14(1)$ & $1 \cdot 1(0 \cdot 4)^{*}$ & $170(14)^{*}$ \\
$10 \mathrm{~d}$ & $17(3)$ & $1 \cdot 2(0 \cdot 3)^{*}$ & $157(20)$ \\
$3 \mathrm{w}$ & $17(4)$ & $0 \cdot 3(0 \cdot 1)$ & $128(21)$ \\
$6 \mathrm{w}$ & $15(3)$ & $0 \cdot 1(0 \cdot 1)$ & $132(18)$ \\
\hline
\end{tabular}

* $p<0.01$ in the comparison with controls.

AM-alveolar macrophages; PMN-polymorphonuclear leucocytes.

room air showed rapid repair of endothelium and epithelium at three days, with type 2 cell proliferation as described previously. ${ }^{12}$ There was little evidence of subsequent fibrosis.

The number of alveolar macrophages recovered by lavage was always in the normal range; an increase in polymorphonuclear leucocytes was seen after six days' exposure only (table 3 ). Glucosaminidase activity was highest on day 6 and was still above normal three days later. The protein concentration in bronchoalveolar lavage fluid corresponded with the morphological evidence of injury. Protein was increased at four days and was more than 10 times normal on day 6 (fig 6). After return to air mice still showed high protein concentrations at three days, after which the values declined to normal. The higher concentration of hydroxyproline in bronchoalveolar lavage fluid indicated that more severe lung injury was produced by prolonged hyperoxia. Hydroxyproline increased after two days and a peak of $0.45 \mu \mathrm{g}$ hydroxyproline/ $\mathrm{ml}$ bronchoalveolar lavage fluid was found at six days (fig 6). No significant change in tissue hydroxyproline was found at any time (fig 3).

\section{Discussion}

The three models of lung injury used in our study are known to produce cell injury and necrosis at the air blood barrier in the initial stages. In many studies the extent of cell injury has been assessed by morphology, or more recently by various measurements on bronchoalveolar lavage fluid. Permeability changes at the blood-air barrier can be evaluated by quantifying protein leakage into the alveoli, whereas cell necrosis is marked by the presence of enzymes such as lactate dehydrogenase. ${ }^{89}$ There have been few studies, however, in which injury to the underlying extracellular matrix has been examined on the basis of bronchoalveolar lavage fluid. Elastolytic activity in bronchoalveolar lavage fluid has been found in patients with the respiratory distress syndrome, ${ }^{15}$ and collagenase has also been found, presumably arising from an efflux of polymorphonuclear leucocytes. ${ }^{8}$ In a recent attempt to use hyperoxia in rats to induce emphysematous changes, Riley et ${ }^{16}{ }^{16}$ could not detect elastin fragments in bronchoalveolar lavage fluid; they did, however, find hydroxyproline in lavage fluid, though not in serum. Many agents that injure the lung act on alveolar cells, so that released products from these cells, or from the extracellular matrix beneath the endothelium and epithelium in particular, can reach the alveolar spaces. Since the alveolar wall is rich in type IV collagen, ${ }^{17}$ damage to the lining cells might result in fragmentation of collagen with deposition of

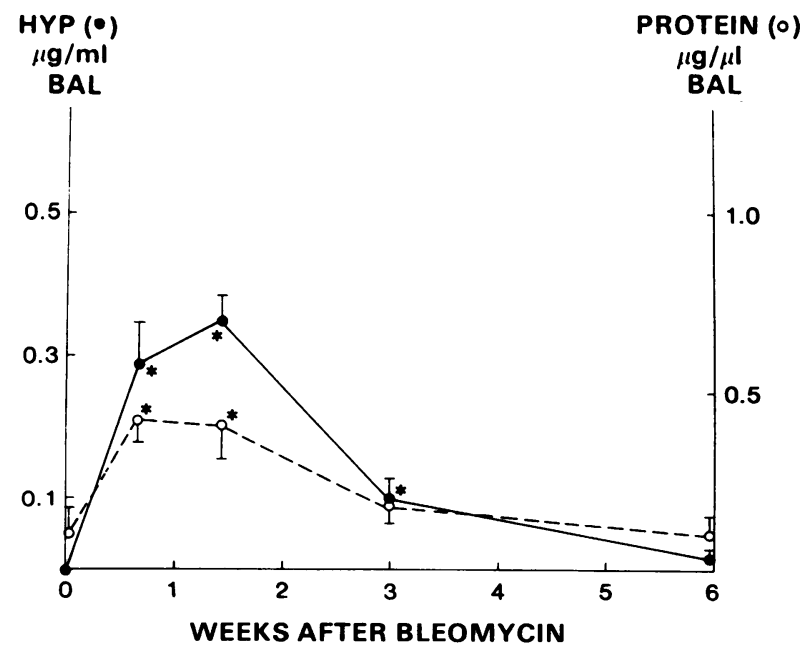

Fig 4 Hydroxyproline (Hyp, closed circles) and protein (open circles) concentrations in lavage (BAL) fluid after bleomycin. * As in figure 2. 


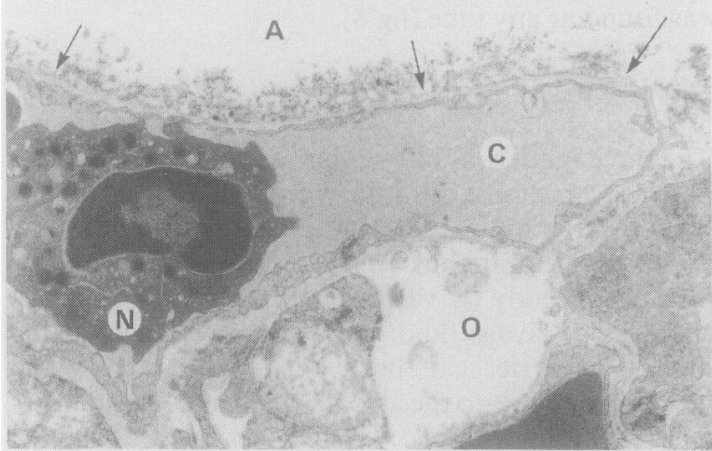

Fig 5 Endothelial injury with interstitial oedema $(O)$ seen after six days' exposure to $95 \%$ oxygen in addition to necrosis of the overlying type I epithelial cells. Cell debris is apparent at the basement membrane of the alveolar $(A)-$ capillary $(C)$ barrier (arrows), and a neutrophil $(N)$ is seen in the capillary.

products in alveolar spaces. In the present study we did not detect any peptide residues containing hydroxyproline in lavage fluid from normal animals. Using different models of acute lung injury we have shown that the level of hydroxyproline in lavage fluid from injured mouse lung increases with the degree of damage to the air-blood barrier as indicated by bronchoalveolar lavage fluid protein.

Radiation induced the lowest level of cell damage and this appeared to be confined to the endothelium.
Table 3 Findings in bronchoalveolar lavage fluid in oxygen experiment (means with standard errors in parentheses)

\begin{tabular}{llll}
\hline Treatment & $\begin{array}{l}A M \\
\left(\times 10^{4}\right)\end{array}$ & $\begin{array}{l}P M N \\
\left(\times 10^{4}\right)\end{array}$ & $\begin{array}{l}\text { Glucosaminidase } \\
(\mu \mathrm{mol} / \mathrm{ml} / \mathrm{h})\end{array}$ \\
\hline [Controls & $15(2)$ & $0 \cdot 1(0 \cdot 1)$ & $115(25)]$ \\
$\mathrm{O}_{2}$ 2 d & $15(3)$ & $0 \cdot 1(0 \cdot 1)$ & $100(10)$ \\
$\mathrm{O}_{2}$ 4 d & $20(4)$ & $0 \cdot 2(0 \cdot 1)$ & $157(11)$ \\
$\mathrm{O}_{2}$ 6 d & $21(4)$ & $1 \cdot 0(0 \cdot 3)^{*}$ & $251(32)^{*}$ \\
$\mathrm{O}_{2}$ 6 d and air 3 d & $16(3)$ & $0 \cdot 2(0 \cdot 2)$ & $221(28)^{*}$ \\
$\mathrm{O}_{2}$ 6 d and air 2 w & $18(3)$ & $0 \cdot 1(0 \cdot 1)$ & $109(10)$ \\
$\mathrm{O}_{2}$ 6 d and air 6 w & $17(2)$ & $0 \cdot 1(0 \cdot 1)$ & $111(11)$ \\
\hline
\end{tabular}

*p $<0.01$ in the comparison with controls.

AM-alveolar macrophages; PMN-polymorphonuclear leucocytes.

This resulted in increased permeability, as shown by protein leakage, at the time when hydroxyproline was detectable in lavage fluid. Bleomycin produced more substantial injury to the endothelium and alveolar epithelium after five and 10 days. Higher concentrations of alveolar protein were found and hydroxyproline was significantly increased at these times. At the time when cell regeneration is known to occur (three weeks), and later when fibrosis develops, ${ }^{1}$ the endothelial and epithelial cells appeared intact and there was no further vascular leakage or collagen breakdown.

The most severe injury seen in this study was produced by six days' hyperoxia. This followed endothelial injury, increased vascular permeability and increased hydroxyproline in lavage fluid. On day 6 some animals died of diffuse alveolar injury and solid lungs; others were in distress and showed high protein

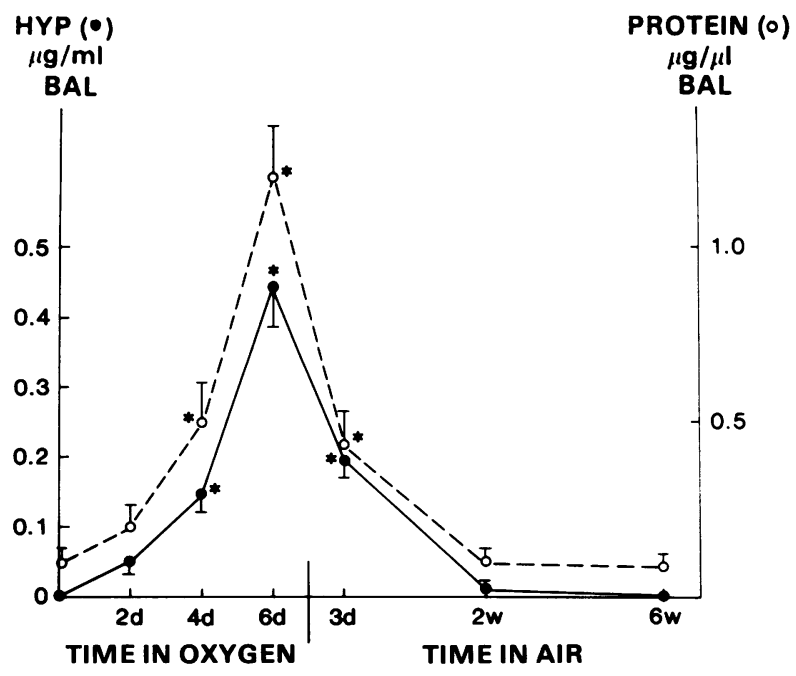

Fig 6 Hydroxyproline (Hyp, closed circles) and protein (open circles) concentrations in lavage (BAL) fluid after hyperoxia. * As in figure 2. 
concentrations with widespread type I cell necrosis. These animals had the highest concentration of hydroxyproline in lavage fluid.

From the combined results of these experiments it appears that endothelial necrosis alone can cause collagen breakdown, with detectable fragments in bronchoalveolar lavage fluid, though more extensive collagen destruction occurs with the combination of endothelial injury and type I epithelial cell necrosis.

Possibly hydroxyproline containing proteins other than collagen could contribute to the raised hydroxyproline concentrations in lavage fluid. There are small amounts of hydroxyproline at the alveolar surface associated with the apoprotein of surfactant and with complement; hydroxyproline could not, however, be detected in lavage fluid from normal lung. In addition, after bleomycin injection the acute phase of injury is associated with substantial alveolar protein leakage and no change in surfactant concentrations, whereas the surfactant lipids doubled at a later stage, most likely as a result of epithelial proliferation when protein concentrations were returning to normal. ${ }^{18}$ These data suggest that the hydroxyproline found here during acute lung injury is not due to changes in surfactant apoprotein concentrations. Moreover, the complement component Clq contains a collagen like helix, but this does not result in detectable hydroxyproline concentrations in normal lavage fluid, or in serum..$^{16}$ In addition, the Clq molecule is very large ( $\mathrm{mw}>450000$ daltons) and is less likely to leak into the alveoli than are the smaller plasma proteins such as albumin that make up the bulk of bronchoalveolar lavage fluid protein in oedema. As the major protein containing hydroxyproline is collagen, probably the hydroxyproline found in lavage fluid originated from the extracellular matrix of the lung.

Increasing amounts of hydroxyproline were measured in lavage fluid as lung injury progressed but there was no measurable decrease in whole lung hydroxyproline. This supports the argument that the bulk of pulmonary connective tissue in thick interstitial areas was relatively unaffected, whereas collagen beneath the focally injured cells of the airblood interface was damaged. This change in the alveolar wall component does not appear to be sufficient to cause a significant decrease in total lung collagen, but none the less is detectable in bronchoalveolar lavage fluid as the collagen fragments collect in the alveolar pool. Any collagen fragments leaking into the circulation are probably diluted out so that hydroxyproline is not detectable in serum during lung injury. ${ }^{16}$ Fragments are also more likely to be carried to the alveolar spaces with the exuded plasma proteins at times of increased permeability. Although up to $40 \%$ of new collagen is rapidly degraded, ${ }^{19}$ no hydroxyproline was detectable in bronchoalveolar lavage fluid from normal mice and, when rates of collagen synthesis were highest at later times after bleomycin injection and irradiation, hydroxyproline concentrations in lavage were again very low. This suggests that the increasing amount of hydroxyproline in bronchoalveolar lavage fluid in the acute injury phase was not due to increased collagen turnover.

The most likely explanation for the presence of hydroxyproline in the alveoli is collagen breakdown of the air-blood barrier, though the exact mechanism for this is unknown. Since type IV collagen is synthesised by endothelial and epithelial cells, the collagenous component of the extracellular matrix may be affected by the necrosis of the overlying cells in these models of injury. It is also possible that a direct free radical reaction degrades the collagenous component of the matrix. Free radicals are produced by hyperoxia, irradiation, and bleomycin, and direct collagen breakdown by free radicals has been found in vitro. ${ }^{19-21}$ It has also been proposed that the products of polymorphonuclear leucocytes may cause tissue destruction. Higher concentrations of hydroxyproline in lavage fluid were associated with increased glucosaminidase activity, which originates predominantly from polymorphonuclear leucocytes. Collagen degradation by enzymes released by polymorphonuclear leucocytes could occur in each case, though few leucocytes were seen in the lung capillaries or in lavage fluid after four days of hyperoxia or after bleomycin injection. Oxygen radicals from polymorphonuclear leucocytes, however, probably play a part in the most severely injured lung after six days' hyperoxia, when sequestered polymorphonuclear leucocytes were seen in capillaries and hydroxyproline concentrations were maximal in the lavage fluid. Interestingly, hydroxyproline was increased in lavage fluid after irradiation when very few polymorphonuclear leucocytes were present in the circulation, and this occurred in animals receiving chlortetracycline, which is known to inhibit collagenase activity. ${ }^{22}$ In this case, direct collagen degradation by free radicals may be the more likely mechanism. It is suggested that the hydroxyproline found in lavage fluid in various examples of lung injury is probably the result of combined free radical and enzymatic degradation of collagen.

This study indicates that collagen breakdown occurs after endothelial and type I cell necrosis in the lung, and that bronchoalveolar lavage is a useful method for investigating this aspect of lung injury. High hydroxyproline concentrations in bronchoalveolar lavage fluid suggest a more severe type of lung damage than a pure permeability change. In general, cellular behaviour varies with the composition of the underlying substrate,${ }^{46}$ so that any alteration in the collagenous component of the lung during 
acute cell injury could influence the proliferation, differentiation, and function of the surviving cells that must regenerate during alveolar repair.

This research was supported by grants from the Medical Research Council of Canada and the Council for Tobacco Research, USA, Inc.

\section{References}

1 Bowden DH. Alveolar response to injury. Thorax 1981;36:801-4.

2 Adamson IYR, Bowden DH. The type 2 cell as progenitor of alveolar epithelial regeneration. Lab Invest 1974;30:35-42.

3 Bowden DH, Adamson IYR. Endothelial regeneration as a marker of the differential vascular response in oxygen-induced pulmonary edema. Lab Invest 1974;30:350-7.

4 Kleinman HK, Klebe RJ, Martin GR. Role of collagenous matrices in the adhesion and growth of cells. J Cell Biol 1981;88:473-85.

5 Madri JA, Williams SK. Capillary endothelial cell cultures: phenotypic modulation by matrix components. J Cell Biol 1983;97:153-65.

6 Lee EY, Parry G, Bissell MJ. Modulation of secreted proteins of mouse mammary epithelial cells by the collagenous substrata. J Cell Biol 1984;98: 146-55.

7 Haslam PL, Turton CWG, Heard B, et al. Bronchoalveolar lavage in pulmonary fibrosis: comparison of cells obtained with lung biopsy and clinical features. Thorax 1980;35:9-18.

8 Reynolds HY. Bronchoalveolar lavage. Am Rev Respir Dis 1987;135:250-63.

9 Hay JG, Haslam PL, Dewar A, Addis B, TurnerWarwick M, Laurent GJ. Development of acute lung injury after the combination of intravenous bleomycin and exposure to hyperoxia in rats. Thorax 1987;42:374-82.

10 Adamson IYR, Bowden DH. Endothelial injury and repair in radiation-induced pulmonary fibrosis. $A m J$ Pathol 1983;112:224-30.
11 Adamson IYR. Drug-induced pulmonary fibrosis. Environ Health Perspec 1984;55:25-36.

12 Lowry OH, Rosebrough NJ, Farr AL, Randall RJ. of Protein measurement with the folin-phenol reagent. Eur J Biol Chem 1951;193:265-75.

13 Bossman HB, Lockwood T, Morgan HR. Surface biochemical changes accompanying primary infection with Rous sarcoma virus. II Proteolytic and glycosidase activity and sublethal autolysis. Exp Cell Res 1974;83:25-30.

14 Woessner JF. Determination of hydroxyproline in connective tissue. In: Hall DA, ed. The methodology of $\bar{\omega}$ connective tissue research. Oxford: Joynson and Bruvvers, 1976:227-33.

15 Lee CT, Fein AM, Lippmann M, Holtzman H, Kimbel P, Weinbaum G. Elastolytic activity in pulmonary lavage fluid from patients with adult respiratory distress syndrome. N Engl J Med 1981;304:192-6.

16 Riley DJ, Kramer MJ, Kerr JS, Chae CV, Yu SY, Berg RA. Damage and repair to lung connective tissue in rats exposed to toxic levels of oxygen. Am Rev Respir Dis 1987;135:441-7.

17 Madri JA, Furthmayr H. Collagen polymorphism in the lung. An immunochemical study of pulmonary fibrosis. Hum Pathol 1980;11:353-66.

18 Thrall RS, Swendsen CL, Shannon TH, et al. Correlation of changes in pulmonary surfactant phospholipids with compliance in bleomycin-induced pulmonary fibrosis in the rat. Am Rev Respir Dis 1987;136:113-8.

19 Bienkowski RS, Cowan MJ, McDonald JA, Crystal RG. Degradation of newly synthesized collagen. $J$ Biol Chem 1978;253:4356-63.

20 Monboisse JC, Braquet P, Randoux A, Borel JP. Nonenzymatic degradation of acid soluble calf skin collagen by superoxidion: protective effect of flavonoids. Biochem Pharmacol 1983;32:53-8.

21 Venhatasubramanian K, Joseph KT. Action of singlet oxygen on collagen. Indian $J$ Biochem Biophys 1977;14:217-20.

22 Golub LM, Wolff M, Lee HM, et al. Further evidence that tetracyclines inhibit collagenase activity in human crevicular fluid and from other mammalian sources. $J$ Peridont Res 1985;20:12-23. 